\title{
Changes and challenges: a career in prostate surgery
}

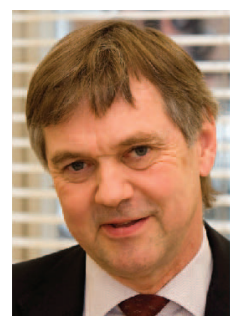

"From the start, when all we had was castration and radiotherapy, to now, when we have robot surgery, robot radiotherapy and all of these emerging new drugs, the outlook for patients has never been better."

Roger Kirby

The Prostate Centre, 32 Wimpole Street, London, W1G 8GT, UK = rkirby@theprostatecentre.com

Roger Kirby speaks to Natasha Galukande, Assistant Commissioning Editor. Roger Kirby graduated in Medical Sciences from Cambridge University (Cambridge, UK) in 1972. He then trained in surgery at University of London (London, UK) before specializing in urology. Kirby is currently the director of The Prostate Centre (London, UK), which he established in 2005. Kirby is the founding editor of the journal Prostate Cancer and Prostate Diseases, in addition to being the associate editor of the British Journal of Urology International, and founding editor of Trends in Urology and Men's Health. He has written over 60 books, published over 300 papers and performed over 2000 radical prostatectomies. In 2005, Kirby was awarded the St Peter's Medal by the British Association of Urological Surgeons. Kirby has also helped to raise over £3 million as trustee and secretary of the Urological Foundation and former chair of Prostate UK.

\section{- What sparked your interest in prostate cancer?}

I trained at Cambridge Medical School, University of Cambridge (Cambridge, UK) and my first job was in Cheltenham General Hospital (Cheltenham, UK). One of the consultants at Cheltenham General Hospital, Peter Boreham, was a urologist. I was inspired by him and by his interest, not just in urology, but particularly in prostate disease. So I began to focus on it and the more I looked into it the more interesting it became. I've basically spent the rest of my life studying it and related diseases.

\section{What do you feel is the biggest challenge facing the field today?}

The main challenge in treating prostate cancer is that we're constantly worrying that we're overtreating some patients and undertreating others. So what we need are tests that give us a better indication of which tumors really need to be treated urgently and which tumors are safe to keep under surveillance for a while. Better molecular prognostic indicators are needed to enable this. These indicators will most likely be genetic, because cancer develops as a result of mutations that alter the DNA. For screening purposes what we need is a better marker than prostate-specific antigen (and perhaps a genetic way of targeting the screening), so you're identifying the one in eight men that are actually going to get prostate cancer, rather than testing the seven out of eight men that are never going to get it.

\section{- After being involved in over 2000 radical prostatectomy operations, how has the focus of these changed \& developed throughout your career?}

Well, I first started doing these in the 1980s when Patrick Walsh from Johns Hopkins (MD, USA) described the nerve sparing, anatomical prostatectomy procedure. After he taught me how to do the operation I came back to England where over time I performed over 1000 open operations. In 2002 the da Vinci $^{\circledR}$ robot came along, which allowed us to undertake robotic prostatectomy. In 2005, I switched from open to robotic and ever since then I have been doing it that way. These developments have had a massive impact on patients and changed my entire working practice. We now have ten-times the magnification and $3 \mathrm{D}$ vision, making the operative field much easier to work with and virtually no blood loss. The whole procedure is now much more a standard laparoscopic procedure rather than open surgery, so there have been huge steps forward.

Learning how to use the da Vinci robot is quite challenging at first. You initially have to have a copilot, it is rather like learning how to fly a helicopter. The first few procedures you do are

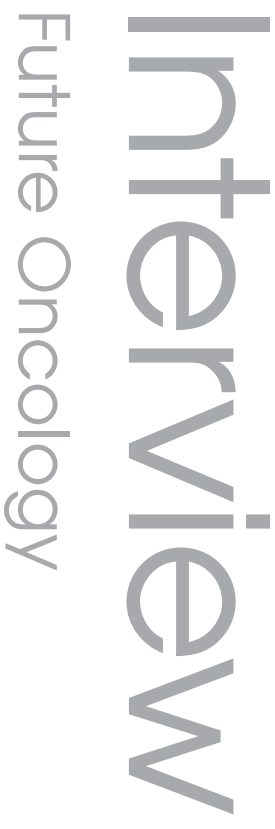

Future $\mathrm{fSS}$ 
quite scary, for the operator and for the patient. But once you get through that the learning curve is quite steep. I've now completed more than 1000 robot-assisted operations, so I really know what I'm doing.

\section{- What do you feel are the biggest achievements \& greatest challenges of your career so far?}

I think doing over 2000 radical prostatectomy operations, 1000 of which were robotic, is a great achievement. Particularly since I had to learn a new minimally invasive technique at the age of 55 years. I'm also quite proud of founding the prostate and urology foundation charities; getting them off the ground and helping to create Prostate Cancer UK, which now raises almost $£ 30$ million per annum. At first both charities were controversial, because when you start something up, it is quite challenging to get people to donate to a new charity. Once it's established it's a lot easier, but getting it started is the hardest step. I also set up the journal Prostate Cancer and Prostate Diseases, which was also quite a tough assignment. When you start a journal it's quite hard as there is no track record and no one wants to submit anything when there are plenty of other rival more established journals where they can publish. So you have to write to all your friends and say 'come on, send me your papers'.

- As the director \& founder of The Prostate Centre, can you tell our readers what inspired you to establish this \& how it has developed over the last 8 years?

We started it in 2005, prior to this I had worked in the UK NHS for 25 years (sometimes I joke that that's longer then you get for murdering your wife!). You can't do something like create a prostate center with a men's health agenda in the NHS because it's too innovative a move. So we decided to set it up as a center of excellence outside the NHS. We found an excellent building at 32 Wimpole Street (London, UK) and we got some great colleagues to join us. It's been very rewarding as we've now looked after many thousands of patients, I think we give a good service and we've developed a men's health agenda, not just in prostate diseases. We have a basic belief that it's no good just looking out for the prostate, or prostate cancer, you've got a patient to look after. So we do prostate specialization with a men's health component. Developments in The Prostate Centre have been established in conjunction with the da Vinci robot. We've seen MRI develop and get better at targeting where the cancer is, new genetic markers for prostate cancer that are now coming to the forefront, and emerging drug treatments such as abiraterone and enzalutamide, which are changing the face of prostate cancer and improving survival. There have been all sorts of changes over the past 8 years that have shaped the Prostate Centre. Providing the new therapies are evidence-based and better for patients, we will adopt them.

\section{- As the chair of the charity Prostate UK} \& secretary of the British Urological Foundation, you have personally raised over $£ 3$ million through marathon running, mountain trekking \& cycling. Can you tell our readers what these roles entail? How do you balance time between these charity roles \& your career?

We have climbed up Mount Kilimanjaro (Tanzania, east Africa) and Mount Kinabalu (Sabah, Borneo), trekked in the Himalayas, Patagonia and the GR20, a very arduous trek down the spine of Corsica. We've done cycling challenges in Sicily, Malawi and Madagascar. We usually go away for a week; into the wilderness and into the mountains. If we are cycling we go off roads and on roads. We aim to raise approximately $£ 0.25$ million, sometimes $£ 0.5$ million, with each one we do. So if you add up all of the previous sponsorship then that comes to over $\mathfrak{3}$ million.

I have a busy schedule at work but it's quite nice to take a break from that, so actually although it is hard physical work, it is mentally relaxing to do something different like this. We have a core group who come on most of these adventures, a patient called Andrew Etherington has come on nearly all of them. He had his prostate removed so he wants to support the cause by coming along with us. There are also doctors and patients from all over; whoever's available and whoever wants a challenge.

In November 2013 we are cycling round the cape of South Africa for The Urology Foundation [1]. It is about $430 \mathrm{~km}$ in 5 days and at the moment there are about 25 people signed up. It is good fun and they are a cheery group with a wide age range; some young ones like my son who is 25 years old and Andrew who is 75 years old.

- You attend many international scientific meetings \& lectures across the globe. Can you tell our readers what you enjoy about these international collaborations \& what the benefits are?

I've just come back from a meeting of the American Association of Genitourinary Surgeons (AAGUS; LA, USA), it included 150 top 
surgeons in the world networking, learning and socializing, which is great. I also regularly attend the American Urological Association (AUA) with approximately 18,000 other people. I know quite a few people around the world in urology so it's a good opportunity to meet up with them. In the smaller meetings there's a more elite group in attendance and you get the chance to meet more people than is the case in the bigger meetings. The Annual Meeting of British Urologists (Manchester, UK) is always fun; it's like a band of brothers as most British urologists know each other. I like to perform at meetings, rather then just attend; it's fun and a bit of a challenge. You also have to go to meetings prepared to learn; things are moving so fast in urology. Although I'm over the age of 60 years, I have to be prepared to not just teach, but to also be taught and continue to learn.

\section{Are you currently involved in any research?}

I used to undertake research as a Professor at $\mathrm{St}$ Georges (London, UK) and St Bartholomew's (London, UK), where we had our own research departments. Since setting up The Prostate Centre I now raise money for other people to do research. Through Prostate Cancer UK we're now raising more than $£ 25$ million each year. By fundraising I can help them to support a lot of research at the University of Cambridge, Oxford University (Oxford, UK), Imperial (London, UK) and University College London (London, UK), for example. I enjoy taking this step back and raising money for other people to do research. I think research is mainly for younger people and most major breakthroughs are made by younger people. Research is hard work and you need lots of brilliant ideas. A more senior person like myself has lots of contacts that I can network with, so that is my main contribution to research these days.

\section{What are the current 'hot topics' in research?}

Nearly 70 genes have been identified as prostate cancer susceptibility genes. So we need to figure out what each of those genes do and how they play a role in developing prostate cancer. We need to improve imaging techniques; MRI is looking very promising but there's more work to be done to get better images, making targeted abnormalities within the prostate possible. Robotic surgery is great but there are other less invasive ways of dealing with prostate cancer, such as better radiotherapy. Hormone therapy works for a while but then fails, so new drugs, such as abiraterone and enzalutamide, are exciting chemotherapy options when combined with docetaxel and cabazitaxel. There's also a whole load of new smart drugs coming down the line that inhibit specific points of the prostate cancer pathway; such as angiogenesis inhibitors and tyrosine kinase inhibitors. These smart new anticancer drugs will improve not only the person's life expectancy and their overall survival, but also their quality of life. Prostate cancer will turn from being a life threatening disease to a chronic disease because we'll be able to manage it more effectively.

\section{- Finally, what progress do you think will be made in treatment in the next 10 years?}

I think better and more accurate diagnosis and staging, kinder and gentler surgical and radiotherapy treatments, and new drugs that are targeted to specific parts of the cell cycle will be developed. Genetic targeting for screening, more accurate prognostic indicators, partial prostatectomy rather than complete removal and new treatment options that are less invasive will be developed. More accurate radiotherapy from CyberKnife ${ }^{\circledR}$ type robotic radiotherapy technology and better treatments for hormone-escape prostate cancer with new treatments will prolong survival. In my 30 years studying prostate cancer I've seen many incredible developments. From the start, when all we had was castration and radiotherapy, to now, when we have robot surgery, robot radiotherapy and all of these emerging new drugs, the outlook for patients has never been better. But there are all sorts of interesting prospects for the next 10 years; therefore, I'm optimistic about the future.

\section{Disclaimer \\ The opinions expressed in this interview are those of the interviewee and do not necessarily reflect the views of Future Medicine Ltd. \\ Financial \& competing interests disclosure \\ $R$ Kirby has no relevant affiliations or financial involvement with any organization or entity with a financial interest in or financial conflict with the sub- ject matter or materials discussed in the manuscript. This includes employment, consultancies, honoraria, stock ownership or options, expert testimony, grants or patents received or pending, or royalties. \\ No writing assistance was utilized in the production of this manuscript.}

\section{Website}

1. The Urology Foundation. www.theurologyfoundation.org 